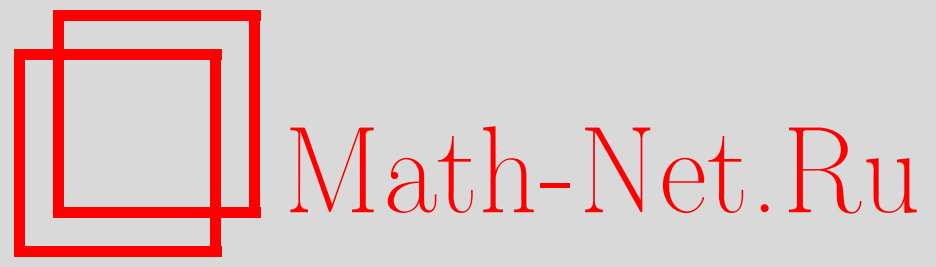

А. А. Досиев, Голоморфные функции от базиса нильпотентной алгебры Ли, Функи. анализ и его прил., 2000, том 34, выпуск 4, 82-84

DOI: https://doi.org/10.4213/faa330

Использование Общероссийского математического портала MathNet.Ru подразумевает, что вы прочитали и согласны с пользовательским соглашением

http://www. mathnet.ru/rus/agreement

Параметры загрузки:

IP: 54.147 .182 .235

26 апреля 2023 г., 17:55:01

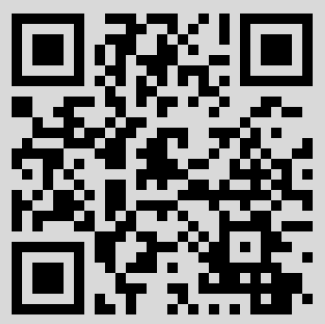




\title{
Голоморфные функции от базиса нильпотентной алгебры Ли
}

\author{
(C) 2000. А. А. ДОСиЕВ
}

В работе [1] Дж. Тейлором предложена общая схема построения функционального исчисления, в частности включающая в себя процедуру пополнения универсальной обертывающей алгебры $\mathscr{U}(E)$ конечномерной алгебры Ли $E$. Задача о функциональном исчислении для заданного гомоморфизма $\alpha: \mathscr{U}(E) \rightarrow A$ в произвольную банахову алгебру $A$ относительно некоторого семейства топологических алгебр «функций» $\mathscr{O}_{\gamma}$ и гомоморфизмов $\varphi_{\gamma}: \mathscr{U}(E) \rightarrow \mathscr{O}_{\gamma}$ формулируется как вопрос о нахождении тех $\mathscr{O}_{\gamma}$, для которых $\alpha$ продолжается до непрерывного гомоморфизма $\alpha_{\gamma}: \mathscr{O}_{\gamma} \rightarrow A, \alpha_{\gamma} \varphi_{\gamma}=\alpha$. В данной заметке мы введем в рассмотрение топологические алгебры $\mathscr{O}_{E}\left(D_{r}\right)$, которые обобщают алгебры $\mathscr{O}\left(D_{r}\right)$ голоморфных функций на открытых полидисках $D_{r}=D_{r_{1}} \times \cdots \times D_{r_{n}}$ в $\mathbb{C}^{n}$ на случай, когда «координатные функции» являются базисом конечномерной нильпотентной алгебры Ли $E$. Мы устанавливаем при естественных условиях наличие функционального исчисления относительно семейства алгебр $\mathscr{O}_{E}\left(D_{r}\right)$ и свойство отображения спектра (в версии 3. Слодковского [2]) голоморфными функциями некоммутирующих переменных.

1. Если не оговорено противное, всюду далее $E-$ комплексная нильпотентная алгебра Ли с базисом $e=\left(e_{1}, \ldots, e_{n}\right)$, в котором присоединенное представление приводится к строго треугольному виду. Для наборов чисел $s=\left(s_{i}\right)_{i=1}^{n}, t=$ $\left(t_{i}\right)_{i=1}^{n}$ положим $s<t$, если $s_{i}<t_{i}$ для всех $i=1, \ldots, n$; кроме того, положим $s^{-1} \cdot \alpha(e)=\left(s_{1}^{-1} \alpha\left(e_{1}\right), \ldots, s_{n}^{-1} \alpha\left(e_{n}\right)\right)$ для гомоморфизма $\alpha: E \rightarrow A$ и для $s>0$. Пусть $M-$ ограниченное подмножество банаховой алгебры $A,\|M\|=\sup \{\|a\|$ : $a \in M\}, M^{n}=\left\{a_{1} \cdots a_{n}: a_{i} \in M\right\}$ и $\mathrm{SG}(M)=\bigcup_{n \geqslant 1} M^{n}$. Число $\rho(M)=$ $\lim _{n}\left\|M^{n}\right\|^{1 / n}$ называется совместным спектральным радиусом множества $M$ [3].

ЛЕмма 1. Пусть $\mathscr{F}-$ банахова алгебра Ли и $\mathscr{F}_{(1)}-$ замкнутый единичный шар в $\mathscr{F}$. Существуют банахова алгебра $\mathscr{A}(\mathscr{F})$ и непрерывный гомоморфизм $\varphi: \mathscr{F} \rightarrow \mathscr{A}(\mathscr{F})$, такие, ито для любых банаховой алгебры А и непрерывного гомоморфизма $и: \mathscr{F} \rightarrow A$ с ограниченной полугруппой $\operatorname{SG}(u(\mathscr{F}(1)))$ существует единственный непрерывный гомоморфизм $v: \mathscr{A}(\mathscr{F}) \rightarrow A$, такой, ито $v \cdot \varphi=u$.

Ясно, что если $\rho\left(u\left(\mathscr{F}_{(1)}\right)\right)<1$, то полугруппа $\operatorname{SG}\left(u\left(\mathscr{F}_{(1)}\right)\right)$ ограничена. Для любого набора $r>0$ рассмотрим банахову алгебру Ли $E$ с $\ell_{1}$-нормой относительно базиса $r^{-1} \cdot e$. Обозначим получаемую из леммы 1 алгебру $\mathscr{A}(E)$ через $\mathscr{A}\left(r^{-1} \cdot e\right)$. Тогда $\mathscr{U}(E)$ является плотной подалгеброй в $\mathscr{A}\left(r^{-1} \cdot e\right)$, и если $E$ коммутативна, то $\mathscr{A}\left(r^{-1} \cdot e\right)$ - аналитическая банахова алгебра [4, гл. 1], отвечающая набору $r$.

ОПРеДЕЛЕнИЕ 1. Алгеброй голоморфных функций $\mathscr{O}_{E}\left(D_{r}\right)$ на полидиске $D_{r}$ называется пополнение мультинормированной [5, п. 1.2.4] алгебры $\left(\mathscr{U}(E),\|\cdot\|_{s}\right.$, $0<s<r)$, где $\|\cdot\|_{s}-$ норма в $\mathscr{U}(E)$, индуцированная нормой в $\mathscr{A}\left(s^{-1} \cdot e\right)$.

Если $D_{r}=\mathbb{C}^{n}$, то $\mathscr{O}_{E}\left(D_{r}\right)$ является оболочкой Аренса-Майкла [5, п. 5.2.21] алгебры $\mathscr{U}(E)$. Для коммутативной алгебры Ли $E$ алгебра $\mathscr{O}_{E}\left(D_{r}\right)$ совпадает 
с $\mathscr{O}\left(D_{r}\right)$. Можно показать, что любой элемент $f \in \mathscr{O}_{E}\left(D_{r}\right)$ единственным способом разлагается в сходящийся (в топологии алгебры $\mathscr{O}_{E}\left(D_{r}\right)$ ) степенной ряд $f=\sum a_{t_{1} \ldots t_{n}} e_{1}^{t_{1}} \cdots e_{n}^{t_{n}}$. Из этого следует, что любая конечномерная подалгебра Ли в $\mathscr{O}_{E}\left(D_{r}\right)$ является нильпотентной. Пусть $e_{1}, \ldots, e_{m}$ образуют базис дополнительного к коммутанту подпространства алгебры Ли $E$ и $D_{r^{(m)}}=D_{r_{1}} \times \cdots \times D_{r_{m}}$. Рассмотрим радикал Джекобсона $\operatorname{Rad} \mathscr{O}_{E}\left(D_{r}\right)$.

Tеорема 1. Отображение $\pi: \mathscr{O}_{E}\left(D_{r}\right) \rightarrow \mathscr{O}\left(D_{r^{(m)}}\right), \pi\left(\sum a_{i_{1} \ldots i_{n}} e_{1}^{i_{1}} \cdots e_{n}^{i_{n}}\right)=$ $\sum a_{i_{1} \ldots i_{m} 0 \ldots 0} z_{1}^{i_{1}} \cdots z_{m}^{i_{m}}(n-m$ нулей $)$, является непрерывным гомоморфизмом алгебр и $\operatorname{ker}(\pi)=\operatorname{Rad} \mathscr{O}_{E}\left(D_{r}\right)$. При этом $\mathscr{O}_{E}\left(D_{r}\right)$ разлагается в топологическую прямую сумму подпространств $\operatorname{Rad} \mathscr{O}_{E}\left(D_{r}\right) \oplus S$, где $S$ топологически изоморфно алгебре $\mathscr{O}\left(D_{r^{(m)}}\right)$.

Теорема 2. Пусть $A-$ банахова алгебра. Гомоморфизм алгебр Ли $\alpha: E \rightarrow A$ продолжается до непрерывного гомоморфизма алгебр $\alpha: \mathscr{O}_{E}\left(D_{r}\right) \rightarrow A, \alpha(f)$ $=f(\alpha(e))$, в том и только в том случае, когда $\rho\left(r^{-1} \cdot \alpha(e)\right)<1$.

Пусть $\mathscr{X}_{r}(E)$ - множество всех непрерывных мультипликативных линейных функционалов алгебры $\mathscr{O}_{E}\left(D_{r}\right)$ и $\widehat{D}=D_{r(m)} \times\{0\} \subseteq D_{r}$. Если $\lambda \in E^{*}, \lambda(e) \in \widehat{D}$, то $\rho\left(r^{-1} \cdot \lambda(e)\right)<1$ и по теореме $2 \lambda$ продолжается до $\lambda \in \mathscr{X}_{r}(E)$. Таким образом, $\mathscr{X}_{r}(E)=\widehat{D}$.

Определение 1 можно распространить на конечномерные разрешимые алгебры Ли $E$. Отметим, что в ненильпотентном случае канонический гомоморфизм $\mathscr{U}(E) \rightarrow \mathscr{O}_{E}\left(D_{r}\right)$ имеет нетривиальное ядро. Тем не менее теоремы 1 и 2 сохраняют силу и в этом случае.

Пусть теперь $(X, \alpha)$ - банахов $E$-модуль. Если имеет место формула $\rho\left(r^{-1} \cdot \alpha(e)\right)=\max _{i} \rho\left(r_{i}^{-1} \alpha\left(e_{i}\right)\right)$, то условие $\rho\left(r^{-1} \cdot \alpha(e)\right)<1$ в теореме 2 можно заменить условием, что для спектра $\sigma\left(\alpha\left(e_{i}\right)\right)$ имеет место включение $\sigma\left(\alpha\left(e_{i}\right)\right) \subset$ $D_{r_{i}}, 1 \leqslant i \leqslant n$. Эта формула в виде $\rho\left(\left\{T_{1}, \ldots, T_{n}\right\}\right)=\max _{i} \rho\left(T_{i}\right)$ справедлива для триангулируемого множества $\left\{T_{1}, \ldots, T_{n}\right\}$ компактных операторов [6]. Поскольку любая конечномерная разрешимая алгебра Ли компактных операторов триангулируема [7], теорема 2 в этом случае допускает следующее уточнение: если $\alpha(E)$ состоит из компактных операторов, то $\alpha$ продолжается на $\mathscr{O}_{E}\left(D_{r}\right)$ в том и только в том случае, когда $\sigma\left(\alpha\left(e_{i}\right)\right) \subset D_{r_{i}}, 1 \leqslant i \leqslant n$.

2. Пусть $\mathscr{F}-$ конечномерная алгебра Ли и $\Delta(\mathscr{F})-$ множество всех характеров алгебры Ли $\mathscr{F}$ (т. е. всех $\left.\lambda \in \mathscr{F}^{*}, \lambda([\mathscr{F}, \mathscr{F}])=0\right)$. Рассмотрим банахов $\mathscr{F}$-модуль $(X, \alpha)$, где $\alpha: \mathscr{F} \rightarrow B(X)$ - представление алгебр Ли. Для целого $p \geqslant 0$ пусть $C^{p}(\mathscr{F}, X)$ - пространство всех знакопеременных $p$-линейных форм на $\mathscr{F}$ со значениями в $X$. Рассмотрим коцепной комплекс, порожденный $\mathscr{F}$-модулем $\left(X, \alpha_{\lambda}\right)$, где $\alpha_{\lambda}=\alpha-\lambda, \lambda \in \Delta(\mathscr{F})$,

$$
0 \rightarrow X \stackrel{d_{\lambda}^{0}}{\longrightarrow} C^{1}(\mathscr{F}, X) \stackrel{d_{\lambda}^{1}}{\longrightarrow} \cdots \stackrel{d_{\lambda}^{p-1}}{\longrightarrow} C^{p}(\mathscr{F}, X) \stackrel{d_{\lambda}^{p}}{\longrightarrow} \cdots
$$

с дифференциалом

$$
\begin{aligned}
\left(d_{\lambda}^{p} \omega\right)\left(u_{1}, \ldots, u_{p+1}\right)=\sum_{i=1}^{p}( & -1)^{i+1} \alpha_{\lambda}\left(u_{i}\right) \omega\left(u_{1}, \ldots, \widehat{u}_{i}, \ldots, u_{p+1}\right) \\
& +\sum_{i=1}^{p}(-1)^{i+j} \omega\left(\left[u_{i}, u_{j}\right], u_{1}, \ldots, \widehat{u}_{i}, \ldots, \widehat{u}_{j}, \ldots, u_{p+1}\right),
\end{aligned}
$$


где $\omega \in C^{p}(\mathscr{F}, X)$, а $\widehat{u}_{i}$ означает пропуск переменной $u_{i}$. Если $H^{p}(\lambda)$ есть $p$ е (преднормированное) пространство когомологий этого комплекса, то положим $\Sigma_{p}(\alpha)=\left\{\lambda \in \Delta(\mathscr{F}): H^{p}(\lambda) \neq\{0\}\right\}, \quad \Sigma_{p}^{\mathrm{e}}(\alpha)=\left\{\lambda \in \Delta(\mathscr{F}): \operatorname{dim} H^{p}(\lambda)=\infty\right\}$. Пусть $\sigma_{\delta, k}(\alpha)=\bigcup_{n-k \leqslant p \leqslant n} \Sigma_{p}(\alpha)\left(\sigma_{\delta, k}^{\mathrm{e}}(\alpha)=\bigcup_{n-k \leqslant p \leqslant n} \Sigma_{p}^{\mathrm{e}}(\alpha)\right)$, и пусть $\sigma_{\pi, k}(\alpha)$ $\left(\sigma_{\pi, k}^{\mathrm{e}}(\alpha)\right)$ есть множество всех $\lambda \in \Delta(\mathscr{F})$, для которых $\lambda \in \bigcup_{0 \leqslant p \leqslant k} \Sigma_{p}(\alpha)(\lambda \in$ $\left.\bigcup_{0 \leqslant p \leqslant k} \Sigma_{p}^{\mathrm{e}}(\alpha)\right)$ или $H^{k+1}(\lambda)-$ неотделимое пространство, где $0 \leqslant k \leqslant n=$ $\operatorname{dim}(\mathscr{F})$.

ОПРеДЕЛЕНИЕ 2. Совокупность всех $\sigma_{\delta, k}(\alpha)$ и $\sigma_{\pi, k}(\alpha), 0 \leqslant k \leqslant n$, назовем семейством спектров Слодковского представления $\alpha$. Соответственно существенные спектры Слодковского - это совокупность всех $\sigma_{\delta, k}^{\mathrm{e}}(\alpha)$ и $\sigma_{\pi, k}^{\mathrm{e}}(\alpha)$, $0 \leqslant k \leqslant n$.

В дальнейшем $\sigma(\alpha)\left(\sigma^{\mathrm{e}}(\alpha)\right)$ обозначает какой-либо из спектров Слодковского (соответственно существенных спектров Слодковского) представления $\alpha$.

Пусть теперь $(X, \alpha)$ - банахов $E$-модуль и $r>0$ - набор, такой, что $\rho\left(r^{-1}\right.$. $\alpha(e))<1$. По теореме 2 гомоморфизм $\alpha$ продолжается до непрерывного представления $\alpha: \mathscr{O}_{E}\left(D_{r}\right) \rightarrow B(X)$. Пусть $\alpha_{\mathscr{F}}-$ его сужение на подалгебру Ли $\mathscr{F} \subseteq$ $\mathscr{O}_{E}\left(D_{r}\right)$. Если $\lambda \in \sigma(\alpha)$, то по проекционному свойству [8, 9] $\lambda\left(e_{i}\right) \in \sigma\left(\alpha\left(e_{i}\right)\right)$, т. е. $\sigma(\alpha) \subseteq \mathscr{X}_{r}(E)$. Для всякого $M \subseteq \mathscr{X}_{r}(E)$ положим $\left.M\right|_{\mathscr{F}}=\left\{\left.\lambda\right|_{\mathscr{F}}: \lambda \in M\right\}$, где $\left.\lambda\right|_{\mathscr{F}}-$ сужение характера $\lambda$ на $\mathscr{F}$.

Теорема 3. Пусть $\mathscr{F}-$ конечномерная подалгебра Ли в $\mathscr{O}_{E}\left(D_{r}\right),(X, \alpha)-$ банахов Е-модуль и $\rho\left(r^{-1} \cdot \alpha(e)\right)<1$. Тогда

$$
\sigma\left(\alpha_{\mathscr{F}}\right)=\left.\sigma\left(\alpha_{E}\right)\right|_{\mathscr{F}}, \quad \sigma^{\mathrm{e}}\left(\alpha_{\mathscr{F}}\right)=\left.\sigma^{\mathrm{e}}\left(\alpha_{E}\right)\right|_{\mathscr{F}} .
$$

Пусть $f=\left(f_{1}, \ldots, f_{k}\right)$ есть семейство образующих алгебры Ли $\mathscr{F}$ и $\operatorname{sp}(\alpha(f), X)$ $=\bar{f}(\sigma(\alpha)), \operatorname{sp}^{\mathrm{e}}(\alpha(f), X)=\bar{f}\left(\sigma^{\mathrm{e}}(\alpha)\right)$, где $\bar{f}: \Delta(\mathscr{F}) \rightarrow \mathbb{C}^{k}, \bar{f}(\lambda)=\left(\lambda\left(f_{i}\right)\right)_{i=1}^{k}$. Согласно теореме 1 , элементы $f_{i} \in \mathscr{F}$ определяют обычные функции $\pi\left(f_{i}\right)$ на $D_{r_{(m)}}$, которые продолжаются на $D_{r^{(m)}} \times \mathbb{C}^{n-m}$ как постоянные на слое $\mathbb{C}^{n-m}$ функции. Полученные функции обозначим через $\hat{f}_{i}$. Пусть $\hat{f}=\left(\hat{f}_{1}, \ldots, \hat{f}_{k}\right)$.

СледСтвиЕ 1. Если $f=\left(f_{1}, \ldots, f_{k}\right)$ порождает конечномерную подалгебру Ли $в \quad \mathscr{O}_{E}\left(D_{r}\right), \quad m o \operatorname{sp}(f(a), X)=\hat{f}(\operatorname{sp}(a, X)), \operatorname{sp}^{\mathrm{e}}(f(a), X)=\hat{f}\left(\operatorname{sp}^{\mathrm{e}}(a, X)\right)$, где $a=\alpha(e)$.

Автор глубоко признателен Ю. В. Туровскому за постановку задачи и за ценные советы и А. Я. Хелемскому, В. С. Шульману и А. С. Файнштейну за полезное обсуждение работы.

\section{ЛИТЕРАТУРА}

1. Taylor J. Adv. Math., 9, 183-252 (1972). 2. Slodkowski Z. Studia Math., 61, 239-255 (1977). 3. Rota G.-C., Strang W. G. Indag. Math., 22, 379-381 (1960). 4. Грауэрт Г., Реммерт P. Аналитические локальные алгебры. Наука, М., 1988. 5. Хелемский $A$. Я. Банаховы и полинормированные алгебры. Наука, М., 1989. 6. Туровский Ю. В. Функц. анализ и его прил., 18, вып. 2, 77-78 (1984). 7. Ваксман Л. Л., Гурарий Д. Л. Функц. анализ и его прил., 8, вып. 4, 81-82 (1974). 8. Fainstein A. S. J. Operator Theory, 29, 3-27 (1993). 9. Ott C. Pacif. J. Math., 173, 173-179 (1996).

Институт математики и механики АН Азербайджана, отдел «Функциональный анализ» 14 апреля 1999 г. 